\title{
Correction to: Innovative radiation oncology Together-Precise, Personalized, Human
}

\author{
David Krug' ${ }^{1}$ Markus Hecht ${ }^{2}$. Nadja Ebert ${ }^{3}$. Matthias Mäurer ${ }^{4}$ Daniel F. Fleischmann ${ }^{5}$ Emmanouil Fokas ${ }^{6}$. \\ Christoph Straube ${ }^{7,8}$. Nils Henrik Nicolay ${ }^{9}$ Juliane Hörner-Rieber ${ }^{10}$. Daniela Schmitt ${ }^{11}$. Cläre von Neubeck ${ }^{12}$. \\ Constantinos Zamboglou ${ }^{9}$. Elena Sperk ${ }^{13}$. David Kaul ${ }^{14}$. Julia Hess ${ }^{15}$. Stefanie Corradini ${ }^{5}$. Clemens Seidel ${ }^{16}$. \\ Cihan Gani ${ }^{17} \cdot$ Christian Baues $^{18}$ - Benjamin Frey ${ }^{2}$ Oliver Blanck ${ }^{1}$ Tobias Gauer ${ }^{19} \cdot$ Maximilian Niyazi $^{5}$ (ID
}

Published online: 3 November 2021

(c) The Author(s) 2021

\section{Correction to:}

\section{Strahlenther Onkol 2021}

https://doi.org/10.1007/s00066-021-01843-9

The original version of this article unfortunately contained mistakes. The name of the author Hess was corrected. Fig. 1 contained orthographic errors. Additionally, to ensure that the hyperlinks embedded in Fig. 1 can be accessed, Fig. 1 was added as Supplement 3. A reference to Supplement 3 has been added in the description of Fig. 1 in the article.

The original article has been corrected.
Open Access This article is licensed under a Creative Commons Attribution 4.0 International License, which permits use, sharing, adaptation, distribution and reproduction in any medium or format, as long as you give appropriate credit to the original author(s) and the source, provide a link to the Creative Commons licence, and indicate if changes were made. The images or other third party material in this article are included in the article's Creative Commons licence, unless indicated otherwise in a credit line to the material. If material is not included in the article's Creative Commons licence and your intended use is not permitted by statutory regulation or exceeds the permitted use, you will need to obtain permission directly from the copyright holder. To view a copy of this licence, visit http://creativecommons.org/licenses/by/4. $0 /$.

The online version of the original article can be found under https://doi.org/10.1007/s00066-021-01843-9

Prof. Dr. med. Maximilian Niyazi

maximilian.niyazi@med.uni-muenchen.de

Extended author information available on the last page of the article 


\section{Affiliations}

\section{David Krug ${ }^{1} \cdot$ Markus Hecht $^{2} \cdot$ Nadja Ebert $^{3} \cdot$ Matthias Mäurer $^{4}$ - Daniel F. Fleischmann ${ }^{5}$ Emmanouil Fokas $^{6}$. Christoph Straube ${ }^{7,8}$ - Nils Henrik Nicolay ${ }^{9}$ Juliane Hörner-Rieber ${ }^{10}$ • Daniela Schmitt ${ }^{11}$ • Cläre von Neubeck ${ }^{12}$. Constantinos Zamboglou ${ }^{9}$ Elena Sperk ${ }^{13}$. David Kaul ${ }^{14}$. Julia Hess ${ }^{15}$. Stefanie Corradini ${ }^{5}$. Clemens Seidel ${ }^{16}$. Cihan Gani ${ }^{17}$. Christian Baues ${ }^{18}$ • Benjamin Frey² Oliver Blanck ${ }^{1}$ Tobias Gauer ${ }^{19}$ - Maximilian Niyazi ${ }^{5}$ iD}

1 Department of Radiation Oncology, University Hospital Schleswig-Holstein, Kiel, Germany

2 Department of Radiation Oncology, Universitätsklinikum Erlangen, Friedrich-Alexander-Universität Erlangen-Nürnberg, Erlangen, Germany

3 Department of Radiotherapy and Radiation Oncology, Faculty of Medicine and University Hospital Carl Gustav Carus, Technische Universität, Dresden, Germany

4 Department of Radiotherapy and Radiation Oncology, University Hospital, Friedrich-Schiller-University, Jena, Germany

5 Department of Radiation Oncology, University Hospital, LMU Munich, Munich, Germany

6 Department of Radiotherapy and Oncology, University Hospital, Goethe University, Frankfurt, Germany

7 Department of Radiation Oncology, Klinikum rechts der Isar, Technical University of Munich, School of Medicine, Munich, Germany

$8 \quad$ RadioLog, Hof, Germany

9 Department of Radiation Oncology, Medical Center-University of Freiburg, Faculty of Medicine, University of Freiburg, Freiburg, Germany

10 Department of Radiation Oncology, Heidelberg University Hospital, Heidelberg, Germany
11 Department of Radiation Oncology, University Medical Center Göttingen, Göttingen, Germany

12 Department of Particle Therapy, University Hospital Essen, University of Duisburg-Essen, Essen, Germany

13 Department of Radiation Oncology, University Medical Center Mannheim, Medical Faculty Mannheim, Heidelberg University, Mannheim, Germany

14 Department of Radiation Oncology, Charité-Universitätsmedizin Berlin, Corporate Member of Freie Universität Berlin, Humboldt-Universität zu Berlin, Berlin, Germany

15 Research Unit Radiation Cytogenetics, Helmholtz Zentrum München, German Research Center for Environmental Health $\mathrm{GmbH}$, Neuherberg, Germany

16 Klinik für Radioonkologie und Strahlentherapie, Universitätsklinikum Leipzig, Leipzig, Germany

17 Department of Radiation Oncology, Eberhard Karls Universität Tübingen, Tübingen, Germany

18 Department of Radiation Oncology and Cyberknife Center, University Hospital of Cologne, Cologne, Germany

19 Department of Radiotherapy and Oncology, University Medical Center Hamburg-Eppendorf, Hamburg, Germany 